\title{
FUNCTIONS WHOSE DERIVATIVE HAS POSITIVE REAL PART
}

\author{
R. R. LONDON
}

(Communicated by Irwin Kra)

\begin{abstract}
In this paper we find a sharp upper bound for $\left|z f^{\prime}(z) / f(z)\right|$,
\end{abstract} where $f$ is a normalised analytic function with $\operatorname{Re} f^{\prime}(z)>0$ in the unit disc.

Denote by $R$ the class of functions $f$ which are regular in $D=\{z:|z|<1\}$, and satisfy $f(0)=0, f^{\prime}(0)=1, \operatorname{Re} f^{\prime}(z)>0(z \in D)$. In a recent paper [3], D. K. Thomas proved, for some absolute constant $K$, that

$$
\left|\frac{z f^{\prime}(z)}{f(z)}\right| \leq \frac{K}{(1-r) \log (1 /(1-r))} \quad(0<|z|=r<1),
$$

whenever $f \in R$. He also asked what the sharp bound for $z f^{\prime} / f$ might be, and it is this question that prompted the present paper. We prove

THEOREM. Let $f \in R$. Then

$$
\frac{\left|f^{\prime}(z)\right|}{\operatorname{Re} f(z) / z} \leq \frac{1+r}{(1-r)(-1-(2 / r) \log (1-r))} \quad(0<|z|=r<1),
$$

with equality for all $r$ in the case of the function $k \in R$ given by $k(z)=-z-$ $2 \log (1-z)$.

Equality here, for $f=k$, occurs since

$$
k^{\prime}(r)=\frac{1+r}{1-r}, \quad \frac{k(r)}{r}=-1-\frac{2}{r} \log (1-r) \quad(0<r<1),
$$

and it is clear that the upper bound in the theorem is also a sharp upper bound for $\left|z f^{\prime}(z) / f(z)\right|$, whenever $f \in R$.

1. Proof of the theorem. We shall use the following

LEMMA. For $\rho$ and $t$ in $[0,1]$,

$$
-\frac{8}{3} t^{3} \rho+(11 \rho-1) t^{2}+4(1-4 \rho) t+1+\frac{11}{3} \rho \geq 0 .
$$

Proof. Denote the left side of the inequality by $g(\rho, t)$, and the square $[0,1] \times$ $[0,1]$ by $S$. We have

$$
\begin{aligned}
& g(\rho, 0)=1+\frac{11}{3} \rho>0, \quad g(\rho, 1)=4(1-\rho) \geq 0 \quad(0 \leq \rho \leq 1), \\
& g(0, t)=-t^{2}+4 t+1>0, \quad g(1, t)=\frac{2}{3}(1-t)^{2}(7-4 t) \geq 0 \quad(0 \leq t \leq 1),
\end{aligned}
$$

Received by the editors November 11, 1986 and, in revised form, April 8, 1987.

1980 Mathematics Subject Classification (1985 Revision). Primary 30C45. 
so that the minimum value of $g$ on $\partial S$ is zero. Next we consider the critical points $(\rho, t)$ of $g$, given by the equations

$$
\begin{gathered}
8 t^{3}-33 t^{2}+48 t-11=0 \\
\rho\left(4 t^{2}-11 t+8\right)+t-2=0
\end{gathered}
$$

The cubic in (2) is increasing, and so has just one real root $t$, which satisfies $.279 \leq t \leq .28$. The function $(2-t) /\left(4 t^{2}-11 t+8\right)$ is also increasing, and hence $.328 \leq \rho \leq .329$ when $\rho$ is given by (3) and $t$ by (2). By minimising each term of $g$ over these values of $\rho$ and $t$, we obtain $g(\rho, t)>2$. Since the minimum value of $g$ is attained on $\partial S$, or at a critical point of $g$ inside $S$, the proof is now complete.

We can now prove the theorem. Let

$$
k(z)=-z-2 \log (1-z)
$$

then

$$
k^{\prime}(z)=\frac{1+z}{1-z}
$$

which shows that $k \in R$. By putting $z=\rho e^{i \theta}$ and integrating with respect to $\rho$ over $[0, r]$, we obtain

$$
\frac{k(z)}{z}=\frac{1}{r} \int_{0}^{r} \frac{1+\rho e^{i \theta}}{1-\rho e^{i \theta}} d \rho \quad\left(z=r e^{i \theta}\right) .
$$

Now let $f \in R$, then $f^{\prime}$ has a Herglotz representation $[1$, p. 22]

$$
f^{\prime}(z)=\frac{1}{2 \pi} \int_{0}^{2 \pi} \frac{1+e^{-i t} z}{1-e^{-i t} z} d \mu(t)
$$

which gives

$$
\frac{f(z)}{z}=\frac{1}{2 \pi} \int_{0}^{2 \pi} \frac{k\left(z e^{-i t}\right)}{z e^{-i t}} d \mu(t)
$$

using (4), after a similar integration to the one above. Next let

$$
\phi(r)=\max _{|z|=r}\left|\frac{1+z}{1-z}\right| / \operatorname{Re} \frac{k(z)}{z} \quad(0<r<1)
$$

and note that $\phi$ is well defined since $\operatorname{Re}(k(z) / z)>0(z \in D)$ by (4). Using (5) with (7), and then (6), we deduce

$$
\left|f^{\prime}(z)\right| \leq \frac{1}{2 \pi} \int_{0}^{2 \pi}\left|\frac{1+e^{-i t} z}{1-e^{-i t} z}\right| d \mu(t) \leq \frac{\phi(r)}{2 \pi} \int_{0}^{2 \pi} \operatorname{Re} \frac{k\left(z e^{-i t}\right)}{z e^{-i t}} d \mu(t)=\phi(r) \operatorname{Re} \frac{f(z)}{z}
$$

Obviously, equality holds if $f=k$. To complete the proof we need to show that

$$
\phi(r)=\frac{1+r}{1-r} / \frac{k(r)}{r},
$$

and this we shall do by verifying that the maximum in (7) is attained at $z=r$.

We have

$$
\begin{aligned}
& \frac{\partial}{\partial \theta}\left(\left|\frac{1+z}{1-z}\right| / \operatorname{Re} \frac{k(z)}{z}\right) \\
& \quad=\left[\left(\operatorname{Re} \frac{k(z)}{z}\right)\left|\frac{1+z}{1-z}\right|\left(-\operatorname{Im} \frac{2 z}{1-z^{2}}\right)\left|\frac{1+z}{1-z}\right| \frac{\partial}{\partial \theta}\left(\operatorname{Re} \frac{k(z)}{z}\right)\right] /\left(\operatorname{Re} \frac{k(z)}{z}\right)^{2}
\end{aligned}
$$


where $z=r e^{i \theta}$. Now using (4), with the notation

$$
J(r, x)=1-2 r x+r^{2}, \quad x=\cos \theta,
$$

we see that, apart from a nonnegative factor, the right side is

$$
\left[-\left(\int_{0}^{r} \frac{1-\rho^{2}}{J(\rho, x)} d \rho\right)\left(\frac{r}{J(r, x)}+\frac{r}{J(r,-x)}\right)+2 \int_{0}^{r} \frac{\rho\left(1-\rho^{2}\right)}{J^{2}(\rho, x)} d \rho\right](\sin \theta) .
$$

So the maximum in (7) is attained at $z=r$ if the function in square brackets is nonpositive for $-1 \leq x \leq 1,0<r<1$. If we write the corresponding inequality as

$$
\int_{0}^{r} \frac{d}{d t}\left[\int_{0}^{t} \frac{\rho\left(1-\rho^{2}\right)}{J^{2}(\rho, x)} d \rho-\frac{1}{2}\left(\int_{0}^{t} \frac{1-\rho^{2}}{J(\rho, x)} d \rho\right)\left(\frac{t}{J(t, x)}+\frac{t}{J(t,-x)}\right)\right] d t \leq 0,
$$

the integrand is

$$
\frac{1}{2}\left(1-t^{2}\right)\left[\frac{t}{J^{2}(t, x)}-\frac{t}{J(t, x) J(t,-x)}-\left(\frac{1}{J^{2}(t, x)}+\frac{1}{J^{2}(t,-x)}\right) \int_{0}^{t} \frac{1-\rho^{2}}{J(\rho, x)} d \rho\right] \text {. }
$$

Thus it is sufficient to prove

$$
\frac{1}{r} \int_{0}^{r} \frac{1-\rho^{2}}{J(\rho, x)} d \rho \geq \frac{J(r,-x)(J(r,-x)-J(r, x))}{J^{2}(r, x)+J^{2}(r,-x)}=\frac{4 r x J(r,-x)}{J^{2}(r, x)+J^{2}(r,-x)},
$$

where $-1 \leq x \leq 1,0<r<1$. When $x$ is nonpositive this is obvious, so we now assume $0<x \leq 1,0<r<1$.

The last inequality can be written as

$$
\frac{1}{r} \int_{0}^{r} \frac{1-\rho^{2}}{J(\rho, x)} d \rho \geq \frac{1-u}{1+u^{2}}
$$

where $u=J(r, x) / J(r,-x)$, and, since $0<u<1$, it is implied by

$$
\frac{1}{r} \int_{0}^{r} \frac{1-\rho^{2}}{J(\rho, x)} d \rho \geq 1-u=\frac{4 r x}{J(r,-x)} .
$$

Now for $0 \leq \rho \leq x$ we have $0<J(\rho, x) \leq 1-\rho^{2}$, so that

$$
\frac{1}{r} \int_{0}^{r} \frac{1-\rho^{2}}{J(\rho, x)} d \rho \geq 1 \quad(0<r \leq x),
$$

and we have only to prove

$$
\int_{0}^{r} \frac{1-\rho^{2}}{J(\rho, x)} d \rho \geq \frac{4 r^{2} x}{J(r,-x)} \quad(0<x \leq r<1) .
$$

Assume now that $0<x \leq r<1$. We have

$$
\frac{d}{d r}\left(\frac{1}{J(r, x)}\right)=\frac{-2(r-x)}{J^{2}(r, x)} \leq 0,
$$

and by using this with (8) we obtain

$$
\begin{aligned}
\int_{0}^{r} \frac{1-\rho^{2}}{J(\rho, x)} d \rho & \geq x+\int_{x}^{r} \frac{1-\rho^{2}}{J(\rho, x)} d \rho \\
& \geq x+\frac{1}{J(r, x)} \int_{x}^{r}\left(1-\rho^{2}\right) d \rho \\
& =x+\frac{1}{J(r, x)}\left(r-\frac{1}{3} r^{3}-x+\frac{1}{3} x^{3}\right) .
\end{aligned}
$$


So a proof of (9) is reduced to that of

$$
\frac{4 r^{2} x}{J(r,-x)} \leq x+\frac{1}{J(r, x)}\left(r-\frac{1}{3} r^{3}-x+\frac{1}{3} x^{3}\right),
$$

which we observe is true for $x=r$, since $4 r x<J(r,-x)$. The inequality is equivalent to

$$
\text { (10) } \frac{1}{3} r^{5}+\frac{11}{3} x r^{4}-\left(8 x^{2}+\frac{2}{3}\right) r^{3}+\left(\frac{11}{3} x^{3}+x\right) r^{2}+\left(-1+2 x^{2}-\frac{2}{3} x^{4}\right) r-\frac{1}{3} x^{3} \leq 0 \text {, }
$$

and because it is true for $x=r$ it is implied by the inequality

$$
-\frac{8}{3} x^{3} r+\left(11 r^{2}-1\right) x^{2}+\left(4 r-16 r^{3}\right) x+r^{2}+\frac{11}{3} r^{4} \geq 0
$$

in which the left side is the derivative with respect to $x$ of the left side of (10). If we now put $x=t r$, and then $\rho=r^{2}$, this inequality follows from the one in the lemma and the proof is complete.

2. Remarks. A general result of Ruscheweyh [2] has a direct bearing on the problem of maximising $\left|z f^{\prime} / f\right|$ for functions $f$ in $R$. It shows that the extreme function has the form

$$
f^{\prime}(z)=t \frac{1+\alpha z}{1-\alpha z}+(1-t) \frac{1+\beta z}{1-\beta z}
$$

where $|\alpha|=|\beta|=1,0<t \leq 1$. But in this approach the technical details seem rather more awkward than those given here.

Thomas [3] notes from (1) that for bounded functions $f$ in $R$

$$
M\left(r, f^{\prime}\right)=O(1)\left[(1-r) \log \frac{1}{1-r}\right]^{-1}
$$

where $M\left(r, f^{\prime}\right)=\max _{|z|=r}\left|f^{\prime}(z)\right|$. The result of this paper shows that (11) also holds for $f \in R$, whenever $\operatorname{Re}(f(z) / z)$ is bounded.

ACKNOWLEDGements. I am grateful to A. D. Thomas and I. M. Davies for some generously given help during the early stages of this work.

\section{REFERENCES}

1. P. L. Duren, Univalent functions, Springer-Verlag, Berlin and New York, 1983.

2. S. Ruscheweyh, Nichtlineare Extremalprobleme für holomorphe Stieltjesintegrale, Math. Z. 142 (1975), 19-23.

3. D. K. Thomas, On functions whose derivative has positive real part, Proc. Amer. Math. Soc. 98 (1986), 68-70.

Department of MATHEMATiCs and Computer SCience, University College SWANSEA, Singleton PaRK, SWANSEA, SA2 8PP WALES 